\title{
Developing new methods to investigate nuclear physics input to the cosmo- logical lithium problem
}

\author{
K.J. Cook ${ }^{1}$, a , D.H. Luong ${ }^{1}$, E. Williams ${ }^{1}$, I.P. Carter ${ }^{1}$, M. Dasgupta ${ }^{1}$, D.J. Hinde ${ }^{1}$, and K. Ramachandran ${ }^{1, b}$ \\ ${ }^{1}$ Department of Nuclear Physics, \\ Research School of Physics and Engineering, \\ The Australian National University, \\ Canberra, ACT 0200, \\ Australia
}

\begin{abstract}
A significant challenge to nuclear astrophysics is the cosmological lithium problem, where models of Big Bang nucleosynthesis indicate abundances of ${ }^{7} \mathrm{Li}$ two to four times larger than what is inferred via spectroscopic measurements of metal-poor stars. Recent experimental techniques developed for nuclear reaction studies at energies near the fusion barrier, if extended to reactions of astrophysical interest, may help understand nuclear reactions that can affect the production of ${ }^{7} \mathrm{Li}$ during the Big Bang. Experiments at the ANU, using new experimental techniques, have provided complete pictures of the breakup mechanisms of light nuclei in collisions with heavy targets, such as ${ }^{208} \mathrm{~Pb}$ and ${ }^{209} \mathrm{Bi}$ [1]. These experiments revealed dominant breakup mechanisms which had not even been considered in theoretical models. The study of the breakup of ${ }^{6} \mathrm{Li}$ and ${ }^{7} \mathrm{Li}$ following interactions with ${ }^{58,64} \mathrm{Ni}$ and ${ }^{27} \mathrm{Al}$ acts as a stepping stone from this previous work towards future experimental studies of breakup reactions of astrophysical relevance. In all cases studied, breakup is dominantly triggered by nucleon transfer between the colliding partners, but the transfer mechanisms are different. The findings of these experiments and experimental considerations for extensions to reactions of light nuclei, such as $\mathrm{d}+{ }^{7} \mathrm{Be}$, will be presented.
\end{abstract}

\section{The ${ }^{7}$ Li problem}

There is a significant discrepancy between the abundance of ${ }^{7} \mathrm{Li}$ observed in metal-poor halo stars and that predicted by Big Bang Nucleosynthesis (BBN) calculations. On the order of 3-4 times more ${ }^{7} \mathrm{Li}$ is predicted to be present in the primordial universe than is inferred via observation. This disagreement, known as the primordial lithium problem, has posed a significant challenge to astrophysics since its discovery in 1982 [2]. Possible solutions to this discrepancy have come from many areas of physics: i) investigating stellar models [3, 4], ii) improved observations of lithium in metal-poor stars [5], iii) low metallicity gases in the Small Magellanic Cloud [6], iv) non-standard cosmologies and physics beyond the standard model [7-11], and v) nuclear physics input into models. The lack of a satisfactory explanation to this discrepancy has reignited the search for a nuclear physics solution to the primordial lithium problem [12-15].

The search for a nuclear physics solution takes the form of re-examining nuclear physics input into models of BBN. Modern models of BBN are such that they are considered parameter free, depending only on experimentally determined nuclear reaction rates. Shown in figure 1

\footnotetext{
a e-mail: kaitlin.cook@anu.edu.au

bermanent Address: Nuclear Physics Division, Bhabha Atomic Research Centre, Trombay, Mumbai-400085, India
}

is a simplified nuclear reaction network showing the most significant reactions producing ${ }^{7} \mathrm{Li}$ during BBN. Crucially, the dominant production mode for ${ }^{7} \mathrm{Li}$ in $\mathrm{BBN}$ conditions is not direct production through the reaction ${ }^{3} \mathrm{H}(\alpha, \gamma){ }^{7} \mathrm{Li}$ but through the production of ${ }^{7} \mathrm{Be}$ and its subsequent decay through electron capture. Thus, any reaction which reduces the abundance of ${ }^{7} \mathrm{Be}$ will reduce the abundance of ${ }^{7} \mathrm{Li}$ in the early universe. It is here that the search for a nuclear physics solution to the cosmological lithium problem has focused. Of particular interest is the destruction reaction ${ }^{7} \mathrm{Be}(\mathrm{d}, \mathrm{p}) 2 \alpha$.

\section{$2{ }^{7} \operatorname{Be}(\mathrm{d}, \mathrm{p}) 2 \alpha$}

The ${ }^{7} \operatorname{Be}(\mathrm{d}, \mathrm{p}) 2 \alpha$ reaction has been shown in a sensitivity study [17] to be able to resolve the primordial lithium problem if the reaction rate was a factor of 100 times larger at low energies than the previous estimate, which assumed a constant $S$-factor [18]. Later experiments instead found a reaction rate 10 times smaller than the 1972 estimate [19]. However, the experiment by Angulo et. al. was insufficiently sensitive to reactions populating high excitations in ${ }^{8} \mathrm{Be}$. A subsequent search for resonant enhancement of the reaction rate through excited states in ${ }^{9} \mathrm{~B}$ failed to observe such a state [20]. Further theoretical work has placed limits on the resonant destruction of ${ }^{7} \mathrm{Be}$ [14]. 


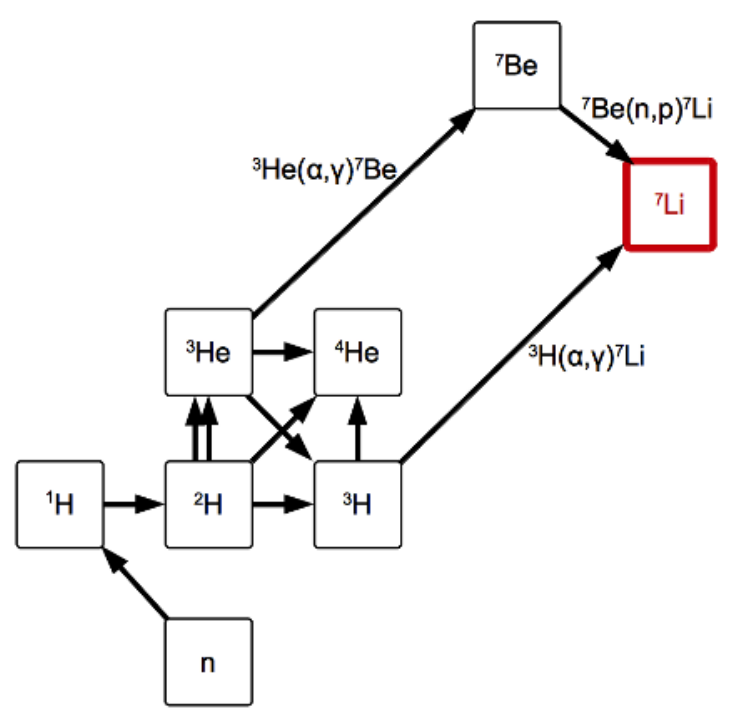

Figure 1. A simplified nuclear reaction network for ${ }^{7} \mathrm{Li}$ production, showing the 12 most important reactions. Adapted from [16]

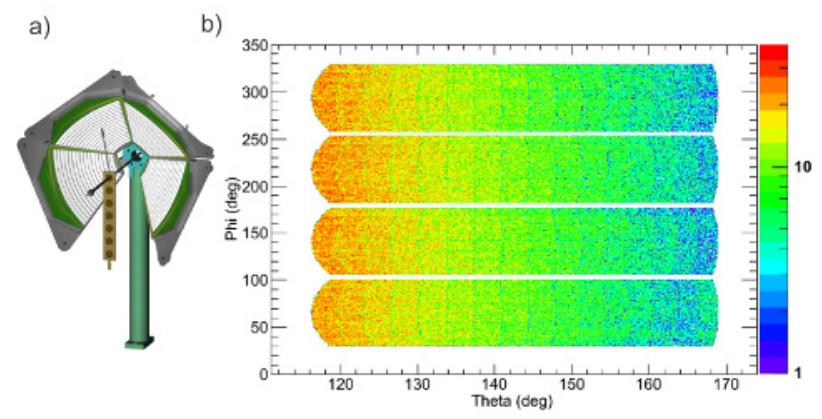

Figure 2. a) A high resolution pixelated detector array comprising four DSSDs mounted on a hub in a "lampshade" annular arrangement all angled at $45^{\circ}$, with respect to their bisector, towards the focal point of the hub. The beam (arrow) and target ladder are also shown. b) The angular coverage, in scattering angle $\theta$ and azimuthal angle $\phi$, of the detector array's 512 pixels for a typical back-angle breakup measurement.

It is therefore apparent that it would be useful to reexamine the ${ }^{7} \mathrm{Be}(\mathrm{d}, \mathrm{p}) 2 \alpha$ reaction rate to further clarify the role of this reaction in the final abundance of ${ }^{7} \mathrm{Li}$ after BBN. A new detector array at the Australian National University (ANU), designed to study the breakup of light nuclei, enables coincident measurements of light charged particles and will allow this re-examination to take place.

\section{The ANU BALIN array}

The ANU Breakup Array for LIght Nuclei (BALIN) consists of four large double sided silicon strip detectors (DSSDs) in a lampshade configuration, as shown in figure 2(a). Each DSSD has sixteen arcs and eight sectors, intersecting to give a highly pixellated array. As such, both

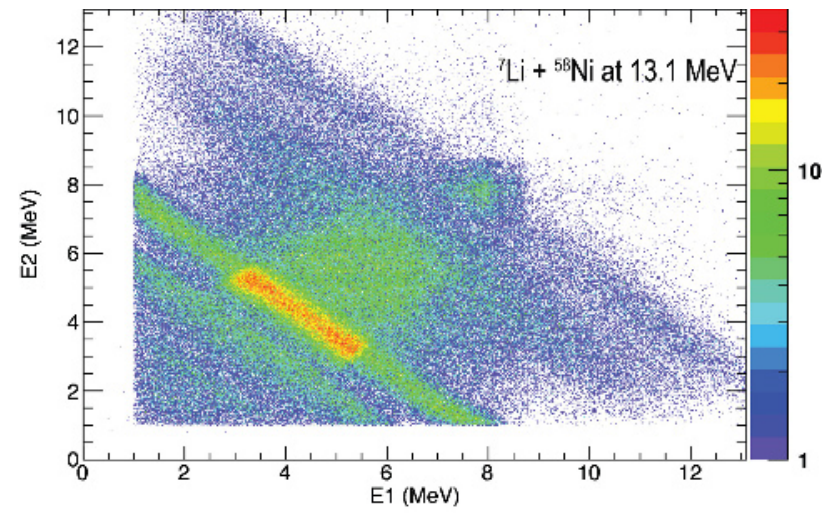

Figure 3. Energy of each particle detected in coincidence, for reactions of ${ }^{7} \mathrm{Li}$ on ${ }^{58} \mathrm{Ni}$ at $13.1 \mathrm{MeV}$. The diagonal groups of events with the same sum total energy $E_{T}=E_{1}+E_{2}$, indicate coincident events originating from the same breakup mode.

position and energy information is recorded for each coincident event, shown in figure 2(b) and figure 3 respectively. Event reconstruction software then enables the determination of two key breakup observables, the Q-value of the reaction and the relative energy, $\mathrm{E}_{r e l}$, of the breakup fragments. The Q-value enables the determination of the state of the target-like recoil nucleus, while the relative energy of the fragments may be used in particular cases to obtain the state of the projectile-like nucleus. It is therefore in principle possible to obtain complete pictures of breakup mechanisms [1].

Previous measurements with this array have been performed examining the breakup of ${ }^{6,7} \mathrm{Li}$ in reactions with very heavy nuclei such as ${ }^{208} \mathrm{~Pb}$ [1], rather far away from the mass of the astrophysically relevant target, deuterium. However, the breakup of ${ }^{6,7} \mathrm{Li}$ on ${ }^{208} \mathrm{~Pb}$ showed the surprising result that transfer induced breakup modes dominated over that of direct breakup, a phenomenon that requires further exploration. There is then a need to examine trends of breakup, and also to simulate the performance of the BALIN array and reconstruction tools with decreasing target mass. Towards this end, measurements were performed to examine the breakup of ${ }^{7} \mathrm{Li}$ after interactions with targets of ${ }^{58} \mathrm{Ni}$ and ${ }^{27} \mathrm{Al}$.

\subsection{Pictures of breakup mechanisms of ${ }^{7} \mathbf{L i}$ reacting with ${ }^{58} \mathrm{Ni}$}

Beams of ${ }^{7} \mathrm{Li}$ were accelerated using the ANU 14UD accelerator at the below-barrier energy of $13.1 \mathrm{MeV}$ onto a ${ }^{58} \mathrm{Ni}$ target with areal density $50 \mu \mathrm{g} \mathrm{cm}^{-2}$, with the charged reaction products detected using the BALIN array. Shown in figure 4 is a scatter plot of the Q-values against the relative energy of coincident events detected in this experiment. The breakup modes of ${ }^{7} \mathrm{Li}$ may be determined through examination of the characteristic Q-values and relative energies apparent in this figure. Horizontal bands of constant Q-value show evidence of breakup events populating the ground and excited states of the target-like recoil 


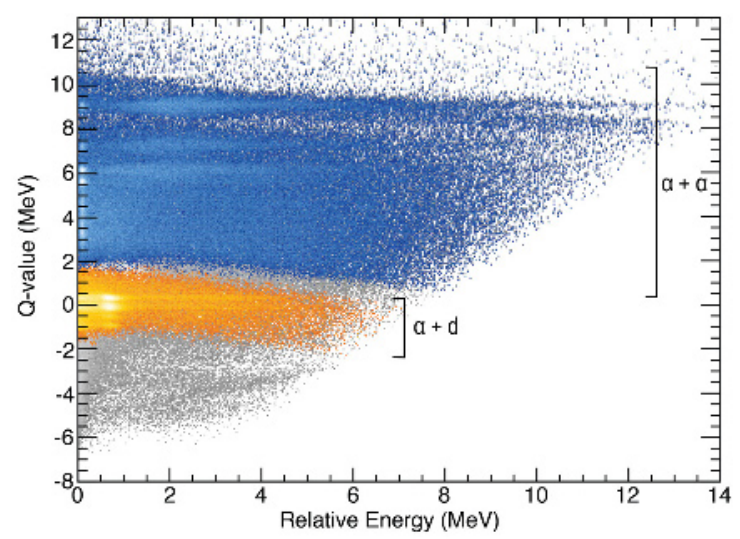

Figure 4. A picture of breakup: Q-value against $\mathrm{E}_{\text {rel }}$ for ${ }^{7} \mathrm{Li}+$ ${ }^{58} \mathrm{Ni}$ at $13.1 \mathrm{MeV}$. Lighter colours show higher intensity. Blue events are those identified as $\alpha+\alpha$ breakup events, while the orange events are those identified as $\alpha+d$ breakup events.

nucleus, while the peaks in relative energy show breakup events populating the ground and excited states of the ejectile nucleus. It can be seen from figure 4 that breakup is essentially exclusively through transfer-induced breakup modes. Peaks in Q-value are seen in the ${ }^{8} \mathrm{Be} \rightarrow 2 \alpha$ identified events (shown in blue) corresponding to events populating the ground and excited states of ${ }^{57} \mathrm{Co}$. The corresponding $\mathrm{E}_{\text {rel }}$ peaks at $92 \mathrm{keV}$ as expected from breakup of ${ }^{8} \mathrm{Be}$ from its ground state, far away from the target-like nucleus, with a broad tail of events extending into high $\mathrm{E}_{\text {rel }}$. This corresponds to breakup and post-acceleration of ${ }^{8} \mathrm{Be}$ in its excited states, close to the target-like nucleus. Neutron stripping from ${ }^{7} \mathrm{Li}$ forming ${ }^{6} \mathrm{Li}$, which breaks up into $\alpha+\mathrm{d}$ events are shown in orange, which have a peak in $E_{\text {rel }}$ corresponding to the breakup of ${ }^{6} \mathrm{Li}$ in its first excited state with expected $E_{\text {rel }}$ of $0.7 \mathrm{MeV}$.

In contrast to previous work in reactions with ${ }^{208} \mathrm{~Pb}$ [1], no evidence of direct breakup of ${ }^{7} \mathrm{Li}$ to $\alpha+t$ was observed.

\subsection{Pictures of breakup mechanisms of ${ }^{7} \mathrm{Li}$ reacting with ${ }^{27} \mathrm{Al}$}

The breakup of ${ }^{7} \mathrm{Li}$ after interactions with ${ }^{27} \mathrm{Al}$ was investigated using a beam of ${ }^{7} \mathrm{Li}$ incident on a ${ }^{27} \mathrm{Al}$ target with areal density $50 \mu \mathrm{g} \mathrm{cm}^{-2}$. Once again, it was seen that the breakup of ${ }^{7} \mathrm{Li}$ was dominated by transfer induced breakup. The dominant modes seen were proton pickup forming ${ }^{8} \mathrm{Be}$ with subsequent $2 \alpha$ breakup, as was seen for ${ }^{7} \mathrm{Li}$ breakup after interactions with ${ }^{58} \mathrm{Ni}$. However, two neutron stripping forming ${ }^{5} \mathrm{Li} \rightarrow \alpha+p$ was also apparent, a breakup mode not seen in reactions with heavier target nuclei. As with reactions of ${ }^{7} \mathrm{Li}$ on ${ }^{58} \mathrm{Ni}$, no direct ${ }^{7} \mathrm{Li}$ $\rightarrow \alpha+t$ breakup was observed.

\section{Towards measurements of the ${ }^{7} \mathrm{Be}(\mathrm{d}, \mathrm{p}) 2 \alpha$ reaction}

The results shown above, whilst demonstrating the dependence of the target species on ${ }^{7} \mathrm{Li}$ breakup, also demonstrate the efficacy of the BALIN array and analysis techniques in measuring the breakup of ${ }^{8} \mathrm{Be}$ in both ground and excited states. These results motivate the use of the array to investigate the astrophysically relevant reaction ${ }^{7} \operatorname{Be}(\mathrm{d}, \mathrm{p}) 2 \alpha$, the experimental considerations for which will be briefly discussed.

To perform this reaction, radioactive ${ }^{7} \mathrm{Be}$ nuclei must be produced. As it has a half life of 53.2 days, it is not particularly suited to production and use as a target. Instead, the reaction may be performed in inverse kinematics, with $\mathrm{a}^{7} \mathrm{Be}$ beam produced off-line via proton irradiation of stable ${ }^{7} \mathrm{Li}$ via the ${ }^{7} \mathrm{Li}(\mathrm{p}, \mathrm{n})^{7} \mathrm{Be}$ reaction and subsequent separation of ${ }^{7} \mathrm{Be}$ from the ${ }^{7} \mathrm{Li}$ bulk using a radiochemical procedure [21]. A deuterium target must therefore be used. Without the facility for gas targets, a thin deuterated polyethylene $\left(\mathrm{C}_{2} \mathrm{D}_{4}\right)_{n}$ target can be used. Such a target has been produced through the dissolution of deuterated polyethylene in xylene, which is then be poured onto glass slides and floated off [22].

To determine the most suitable geometry of the BALIN array for this reaction, Monte Carlo kinematic simulations were performed for the break-up reactions of interest - the simulations take into account only energy and momentum conservation, without taking into consideration any internuclear potential. However, such simulations provide a useful approximation of the distribution of breakup particles in energy and angle. It was found that the best placement of the BALIN array for this reaction is at forward angles, with an angular coverage of approximately $30^{\circ} \leq \theta \leq 80^{\circ}$, a coverage outside the cone of elastically scattered ${ }^{7} \mathrm{Be}$ nuclei on deuterium which are confined to $\theta \leq 16.6^{\circ}$, and one where $\alpha$ particles produced in the breakup of ${ }^{8} \mathrm{Be}$ in its ground and first three excited states have energies above the detection threshold. However, flux from elastically scattered ${ }^{7} \mathrm{Be}$ from the carbon in the $\left(\mathrm{C}_{2} \mathrm{D}_{4}\right)_{n}$ target will be observed with this geometry.

In analogy with the breakup of ${ }^{7} \mathrm{Li}$ on ${ }^{58} \mathrm{Ni}$ shown in figure 3, a scatter plot of the energies of the $\alpha$ particles produced in the reaction ${ }^{7} \mathrm{Be}(\mathrm{d}, \mathrm{p}) 2 \alpha$ at a (laboratory) beam energy of $1.17 \mathrm{MeV}$, half the fusion barrier of this system, is shown in figure 5. Due to the high Q-value of this reaction $(16.8 \mathrm{MeV})$, the expected energies of the $\alpha$ particles produced in this reaction are comparable to the energies of the breakup fragments produced in the reaction ${ }^{7} \mathrm{Li}+$ ${ }^{58} \mathrm{Ni}$ at $13.1 \mathrm{MeV}$. This is further motivation to believe that the BALIN array is well suited to a measurement of this reaction.

\section{Conclusions}

Using the ANU BALIN array, an experimental technique has been successfully established to measure the processes involved in the breakup of light nuclei after interactions with targets from mass 209 down to mass 27 . It was found 


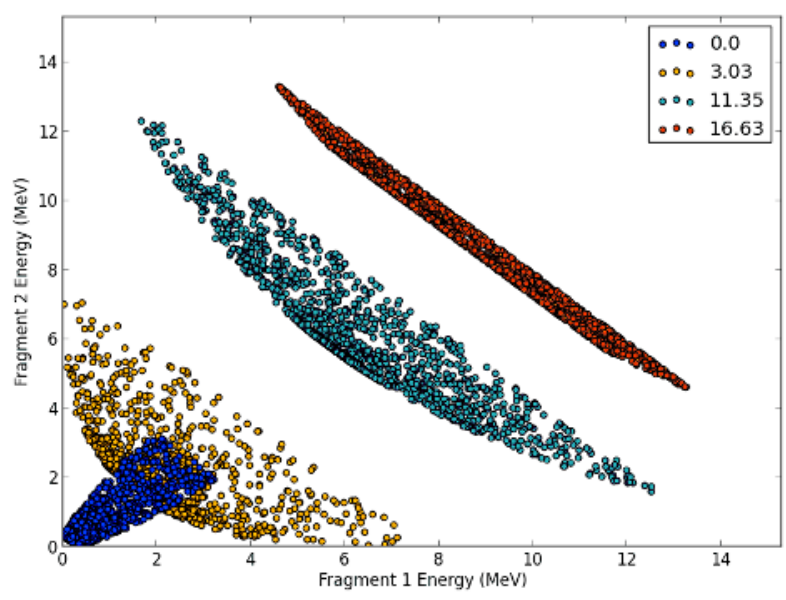

Figure 5. Scatter plot of the energy of the two $\alpha$ particles produced in coincidence in the reaction ${ }^{7} \mathrm{Be}(\mathrm{d}, \mathrm{p}) 2 \alpha$ at a (laboratory) beam energy of $1.17 \mathrm{MeV}$. Colour of points indicates the excitation energy of the projectile-like ${ }^{8} \mathrm{Be}$ nucleus prior to breakup, shown in the legend.

that in reactions with ${ }^{58} \mathrm{Ni}$, the breakup of ${ }^{7} \mathrm{Li}$ is dominated by proton pickup by the projectile leading to the $2 \alpha$ breakup mode, as well as neutron stripping from ${ }^{7} \mathrm{Li}$ producing ${ }^{6} \mathrm{Li}$ and subsequent breakup to the $\alpha+d$ breakup mode. In reactions with ${ }^{27} \mathrm{Al}$, it was found that the breakup modes of ${ }^{7} \mathrm{Li}$ are also dominated by breakup, but these modes are different to those seen after interactions with ${ }^{58} \mathrm{Ni}$ - in this system, breakup again is dominated by proton pickup forming ${ }^{8} \mathrm{Be}$ and subsequently $2 \alpha$; however, two neutron stripping from ${ }^{7} \mathrm{Li}$ was also seen, forming ${ }^{5} \mathrm{Li} \rightarrow \alpha+p$. This reaction mode was not seen in reactions with ${ }^{58} \mathrm{Ni}$ or ${ }^{208} \mathrm{~Pb}$.

Kinematic simulations of the reaction ${ }^{7} \mathrm{Be}(\mathrm{d}, \mathrm{p}) 2 \alpha$ indicate the suitability of the BALIN array for studying this astrophysically relevant reaction.

\section{References}

[1] D.H. Luong, M. Dasgupta, D.J. Hinde, R. Du Rietz, R. Rafiei, C. Lin, M. Evers, A. Diaz-Torres, Physics Letters B 695, 105 (2011)

[2] F. Spite, M. Spite, Astronomy \& Astrophysics 115, 357 (1982)

[3] L. Casagrande, I. Ramírez, J. Meléndez, M. Bessell, M. Asplund, Astronomy \& Astrophysics 512, A54 (2010)

[4] M.H. Pinsonneault, G. Steigman, T. Walker, N. V.K, The Astrophysical Journal 574, 398 (2002)
[5] L. Sbordone, P. Bonifacio, E. Caffau, H.G. Ludwig, N.T. Behara, J.I. González Hernández, M. Steffen, R. Cayrel, B. Freytag, C. Van't Veer et al., Astronomy \& Astrophysics 522 (2010)

[6] J.C. Howk, N. Lehner, B.D. Fields, G.J. Mathews, Nature 489, 121 (2012)

[7] A. Coc, S. Goriely, Y. Xu, M. Saimpert, E. Vangioni, The Astrophysical Journal 744, 158 (2012)

[8] M. Regis, C. Clarkson, General Relativity and Gravitation 44, 567 (2012)

[9] K.A. Olive, P. Petitjean, E. Vangioni, J. Silk, Monthly Notices of the Royal Astronomical Society 426, 1427 (2012)

[10] O. Erken, P. Sikivie, H. Tam, Q. Yang, Physical Review Letters 108, 061304 (2012)

[11] A. Coc, J.P. Uzan, E. Vangioni, ArXiv e-prints (2013), arXiv: $1303.1935 \mathrm{v} 1$

[12] O. Civitarese, M. Mosquera, Nuclear Physics A 898, 1 (2013)

[13] R.H. Cyburt, M. Pospelov, International Journal of Modern Physics E 21, 1250004 (2012)

[14] C. Broggini, L. Canton, G. Fiorentini, F. Villante, arXiv:1202.5232v1 (2012)

[15] G. Gyurky, F. Confortola, H. Costantini, A. Formicola, D. Bemmerer, R. Bonetti, C. Broggini, P. Corvisiero, Z. Elekes, F. Zs et al., Physical Review C 75 (2007)

[16] B. Fields, Annual Review of Nuclear and Particle Science 61, 47 (2011)

[17] A. Coc, E. Vangioni, P. Descouvemont, A. Adahchour, C. Angulo, The Astrophysical Journal 600, 544 (2004)

[18] P.D. Parker, The Astrophysical Journal 175, 261 (1972)

[19] C. Angulo, E. Casarejos, M. Couder, P. Demaret, P. Leleux, F. Vanderbist, A. Coc, J. Kiener, V. Tatischeff, T. Davinson et al., The Astrophysical Journal Letters 630, 105 (2005)

[20] P. O’Malley, D. Bardayan, A. Adekola, S. Ahn, K. Chae, J. Cizewski, S. Graves, M. Howard, K. Jones, R. Kozub et al., Physical Review C 84, 042801 (2011)

[21] L. Gialanella, U. Greife, N.D. Cesare, A. Dõonofrio, M. Romano, L. Campajola, A. Formicola, Z. Fulop, G. Gyurky, G. Imbriani et al., Nuclear Instruments and Methods in Physics Research B 197, 150 (2002)

[22] G.E. Tripard, Review of Scientific Instruments 38, 435 (1967) 